\title{
The Gravitational Radius of an Irradiated Disk
}

\author{
Kurt Liffman ${ }^{1,2}$ \\ ${ }^{1}$ School of Mathematical Sciences, Monash University, Clayton Vic 3168, Australia \\ ${ }^{2}$ Energy and Thermofluids Engineering, CSIRO/MIT, Highett Vic 3190, Australia \\ Kurt.Liffman@csiro.au \\ Received 2003 March 19, accepted 2003 July 5
}

\begin{abstract}
We derive an updated, analytic formula for the gravitational radius, $r_{g}$, of an irradiated, thin disk. For such a disk, the region outside the gravitational radius will produce a thermal wind, while the region interior to the gravitational radius will be stable. We find that $r_{g} \approx 1.4\left(M_{*} / M_{\odot}\right) /\left(T_{0} / 10^{4} \mathrm{~K}\right) \mathrm{AU}$. The value of 1.4 AU is approximately one fifth of currently used values.

The analysis uses the adiabatic approximation. We argue that the same formula applies for the nonadiabatic, isothermal case.
\end{abstract}

Keywords: accretion disks — hydrodynamics — circumstellar matter — stars: winds, outflows

\section{Introduction}

An irradiated, thin disk is stable provided that $r<r_{g}$, where $r$ is a cylindrical coordinate representing the distance from the centre of the star to a point in the disk, and $r_{g}$ is the gravitational radius. The gravitational radius marks the boundary between a static atmosphere and a photoevaporating atmosphere $\left(r>r_{g}\right)$, where it is assumed that the temperature of the upper atmosphere is approximately constant for the $r$ values of interest (Hollenbach et al. 1994).

To determine $r_{g}$, authors equate the sound speed to the Keplerian speed. This gives a good, intuitive derivation for $r_{g}$ given by

$$
r_{g}=\frac{G M_{*} \bar{m}}{k_{B} T_{0}} \approx 6.8 \frac{\left(M_{*} / M_{\odot}\right)}{\left(T_{0} / 10^{4} \mathrm{~K}\right)} \mathrm{AU}
$$

where $k_{B}$ is Boltzmann's constant, $T_{0}$ is the gas temperature at the base of the flow, $\bar{m}$ the mean mass per gas particle, $G$ the universal gravitational constant, and $M_{*}$ the mass of the central star. In this paper, we extend this analysis and suggest that a more appropriate value for $r_{g}$ is

$$
r_{g}=\frac{(\gamma-1)}{2 \gamma} \frac{G M_{*} \bar{m}}{k_{B} T_{0}} \approx 1.4 \frac{\left(M_{*} / M_{\odot}\right)}{\left(T_{0} / 10^{4} \mathrm{~K}\right)} \mathrm{AU}
$$

where $\gamma$ is the ratio of the specific heats (in this case we have assumed a monatomic gas and set $\gamma=5 / 3$ ). The value of $1.4 \mathrm{AU}$ is one fifth of the value given in equation (1).

Equation (2) is consistent with the numerical results of Woods et al. (1996, see their Figure 8) who examined the evaporation of X-ray irradiated disks associated with active galactic nuclei (AGN). Equation (2) is also consistent with the work of Begelman et al. (1983) who showed, theoretically, that thermal winds could arise from $r$ values within the $r_{g}$ value given by equation (1).

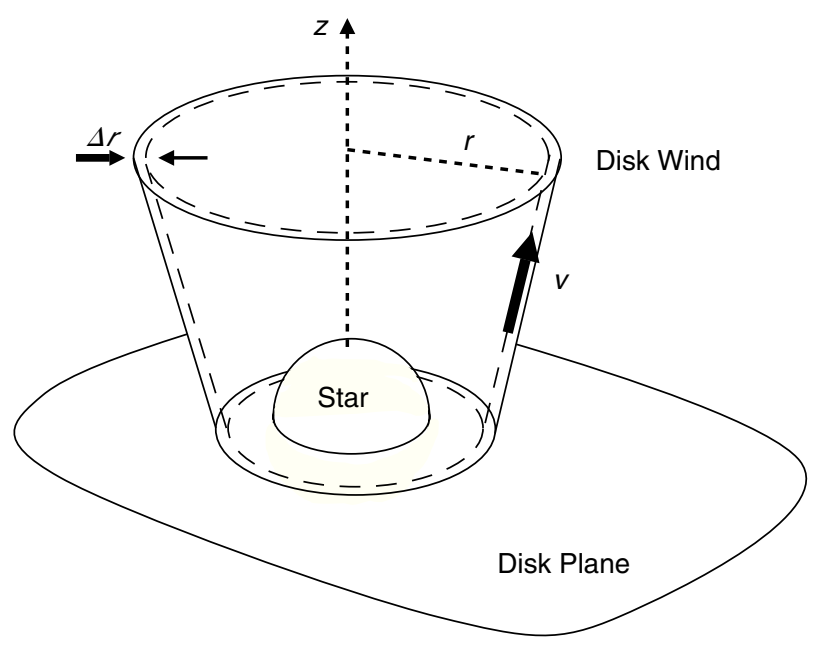

Figure 1 Thermal disk wind.

\section{Thermal Disk Wind}

The hypothetical flow scenario is shown in Figure 1. The wind arises from an unstable section of the disk and expands in a roughly conical shape. We analyse the flow by adopting cylindrical coordinates $(r, \theta, z)$, with the star at the origin, and the $z$ axis perpendicular to the accretion disk.

To simplify the analysis, we assume a steady-state, adiabatic, axisymmetric flow around the $z$ axis.

Given these assumptions our basic equations are: continuity:

$$
\nabla \cdot(\rho v)=0
$$

momentum:

$$
\rho(v \cdot \nabla) v=-\nabla p+\rho g
$$

energy:

$$
\nabla \cdot\left(v\left(p+u+\frac{1}{2} \rho v^{2}+\rho \phi_{g}\right)\right)=0,
$$


where $v$ is the velocity, $u$ the internal energy, $p$ the pressure, $\rho$ the density, and $\phi_{g}$ is the gravitational potential

$$
\phi_{g}=-\frac{G M_{*}}{\sqrt{r^{2}+z^{2}}},
$$

$M_{*}$ being the mass of the central star and $G$ the gravitational constant, and

$$
g=-\nabla \phi_{g}
$$

For a gas, we can assume that

$$
p=(\gamma-1) u,
$$

where $\gamma$ is the ratio of specific heats. Combining equations (8) and (5) implies

$$
\nabla \cdot\left(v\left(\left(\frac{\gamma}{\gamma-1}\right) p+\frac{1}{2} \rho v^{2}+\rho \phi_{g}\right)\right)=0 .
$$

The angular velocity of the flow is readily obtained from the $\theta$ component of equation (4) which, from axisymmetry, is force free, i.e.,

$$
\left.(v \cdot \nabla) v\right|_{\theta}=0,
$$

and has the solution

$$
v_{\theta}(r)=\frac{L}{r},
$$

where $L$, the specific angular momentum, is a constant. For most cases of interest,

$$
L \approx \sqrt{G M_{*} r_{0}},
$$

where $r_{0}$ is the initial value of $r$ when $z \approx 0$.

Using Gauss' Law and noting, from Figure 1, that the normals on the surfaces of our domain of integration are everywhere perpendicular to $\hat{\theta}$, we can write

$$
\int_{S}\left(\frac{1}{2} \rho v^{2}+\left(\frac{\gamma}{\gamma-1}\right) p+\rho \phi_{g}\right) v \cdot d S=0,
$$

where $S$ and $d S$ represent the jet flow surface and an element of that surface, respectively. Because, by construction, only the top and bottom sections of the flow volume contribute non-zero values to the above integral, we have

$$
\left(\frac{1}{2} \rho v^{2}+\left(\frac{\gamma}{\gamma-1}\right) p+\rho \phi_{g}\right) v_{z} r \Delta r=\text { constant }
$$

For equation (14), we assume that the top and bottom sections of the flow volume have $d S=d S \hat{z}$ and that $v_{z}>0$. Similarly, the mass conservation equation (equation (3)) gives

$$
\rho v_{z} r \Delta r=\text { constant } \equiv \mathcal{N}_{1} .
$$

Dividing equation (14) by equation (15) provides us with a Bernoulli equation

$$
\frac{1}{2} v^{2}+\left(\frac{\gamma}{\gamma-1}\right) \frac{p}{\rho}+\phi_{g} \equiv E=\text { constant } .
$$

Here, $E$ is the sum of the (specific) thermal, kinetic, and gravitational energies which is a constant along the flow. Equation (11) implies that

$$
v^{2}=v_{r}^{2}+v_{\theta}^{2}+v_{z}^{2}=v_{p}^{2}+\frac{L^{2}}{r^{2}},
$$

$v_{p}$ being the poloidal velocity component $\left(v_{p}^{2}=v_{r}^{2}+v_{z}^{2}\right)$. The pressure is given by the ideal gas equation

$$
p=\frac{\rho k_{B} T}{\bar{m}},
$$

$k_{B}$ being Boltzmann's constant, $T$ the gas temperature, and $\bar{m}$ the mean mass per gas particle. Combining equations (17), (16), and (6) gives

$$
\frac{1}{2} v_{p}^{2}+\left(\frac{\gamma}{\gamma-1}\right) \frac{p}{\rho}-\frac{G M_{*}}{\sqrt{r^{2}+z^{2}}}+\frac{L^{2}}{2 r^{2}}=E .
$$

Suppose that $v_{p}=0, z \ll r$ and $r=r_{0}$, i.e., the parcel of gas is at or near the surface of a thin disk. Then at this starting point of the flow

$$
E \approx\left(\frac{\gamma}{\gamma-1}\right) \frac{p}{\rho}-\frac{G M_{*}}{2 r_{0}} .
$$

If $E>0$ the system is unbound and a disk wind can form. At $E=0$

$$
\left(\frac{\gamma}{\gamma-1}\right) \frac{p}{\rho}=\frac{G M_{*}}{2 r_{0}}
$$

Combining equations (21) and (18) implies

$$
r_{0}=\frac{(\gamma-1)}{2 \gamma} \frac{G M_{*} \bar{m}}{k_{B} T_{0}} \equiv r_{g},
$$

where $T_{0}$ is the gas temperature at the base of the flow. For a constant $T_{0}, r_{0}>r_{g}$ implies $E>0$ and the system is unbound, while for $r_{0}<r_{g}, E<0$ and the system is bound. For a fully ionised gas, $\bar{m} \approx 1.1 \times 10^{-27} \mathrm{~kg}, \gamma=5 / 3$ (i.e., we assume that the gas is essentially monatomic) and

$$
r_{g} \approx 1.4 \frac{\left(M_{*} / M_{\odot}\right)}{\left(T_{0} / 10^{4} \mathrm{~K}\right)} \mathrm{AU} .
$$

\section{The Non-Adiabatic Case}

Equation (23) is valid for an adiabatic flow from the surface of an accretion disk. For an irradiated disk, however, the flux of extreme ultraviolet photons will tend to keep the coronal gas above the disk at a temperature of $10^{4} \mathrm{~K}$. Thus, one needs to take into account the heat flux in determining the behaviour of the flow. The appropriate modification to the energy equation is

$$
\nabla \cdot\left(v\left(\left(\frac{\gamma}{\gamma-1}\right) p+\frac{1}{2} \rho v^{2}+\rho \phi_{g}\right)\right)=q,
$$

where $q$ is the heat flux and we assume that $q>0$, i.e., heat energy is being added to the system. 


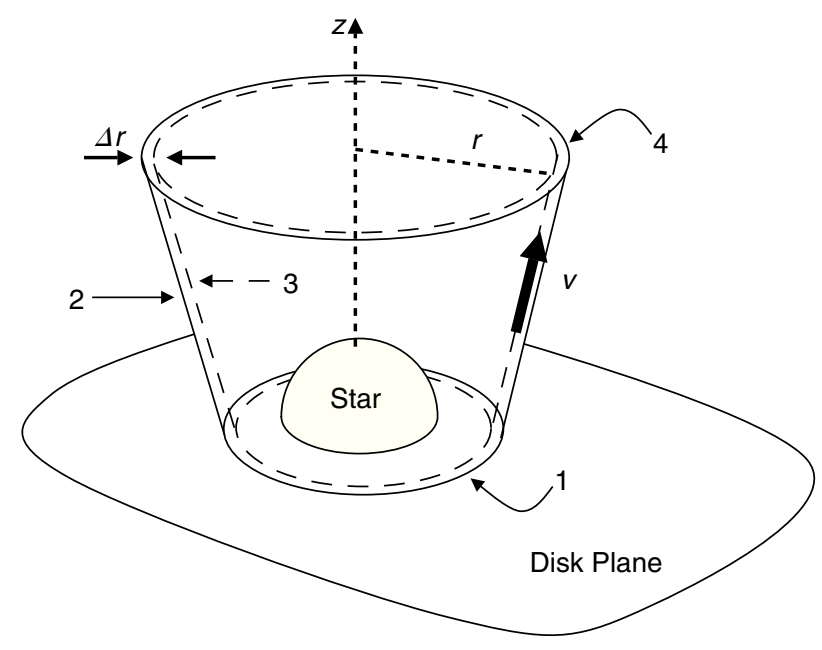

Figure 2 Thermal disk wind with labelled surfaces of the flow volume.

Taking the volume integral of equation (24) and using Gauss' Law implies

$$
\int_{S}\left(\left(\frac{\gamma}{\gamma-1}\right) p+\frac{1}{2} \rho v^{2}+\rho \phi_{g}\right) v \cdot d S=\int_{V} q d V
$$

where $V$ is the integration volume shown in Figure 2. By construction, surfaces 2 and 3 do not contribute to the surface integral, so we can write

$$
\begin{aligned}
& 2 \pi r_{4} \Delta r_{4} v_{z 4}\left(\left(\frac{\gamma}{\gamma-1}\right) p_{4}+\frac{1}{2} \rho_{4} v_{4}^{2}+\rho_{4} \phi_{g 4}\right) \\
= & 2 \pi r_{1} \Delta r_{1} v_{z 1}\left(\left(\frac{\gamma}{\gamma-1}\right) p_{1}+\frac{1}{2} \rho_{1} v_{1}^{2}+\rho_{1} \phi_{g 1}\right) \\
& +\int_{V} q d V
\end{aligned}
$$

Dividing equation (26) by equation (15), we obtain

$$
\begin{aligned}
& \left(\frac{\gamma}{\gamma-1}\right) \frac{p_{4}}{\rho_{4}}+\frac{1}{2} v_{4}^{2}+\phi_{g 4} \\
= & \left(\frac{\gamma}{\gamma-1}\right) \frac{p_{1}}{\rho_{1}}+\frac{1}{2} v_{1}^{2}+\phi_{g 1}+\frac{\int_{V} q d V}{\mathcal{N}_{1}} .
\end{aligned}
$$

We are interested in the surface properties of the flow, as such we let surface 4 approach surface 1 . In the limit, where

$$
\frac{\int_{V} q d V}{\mathcal{N}_{1}} \ll\left(\frac{\gamma}{\gamma-1}\right) \frac{p_{1}}{\rho_{1}}+\frac{1}{2} v_{1}^{2}+\phi_{g 1},
$$

equation (27) approximately approaches equation (16) and the analysis proceeds as for the adiabatic case with the same resulting gravitational radius.
Equation (27) indicates that if $r_{0}>r_{g}$ then

$$
\left(\frac{\gamma}{\gamma-1}\right) \frac{p_{1}}{\rho_{1}}+\frac{1}{2} v_{1}^{2}+\phi_{g 1}>0 .
$$

Since $q>0$ then

$$
\left(\frac{\gamma}{\gamma-1}\right) \frac{p_{4}}{\rho_{4}}+\frac{1}{2} v_{4}^{2}+\phi_{g 4}>0 .
$$

So, once started, the flow will continue to leave the disk.

\section{Conclusion}

By invoking the adiabatic approximation, it is possible to determine the gravitational radius of a flat disk with an isothermal corona:

$$
r_{g}=\frac{(\gamma-1)}{2 \gamma} \frac{G M_{*} \bar{m}}{k_{B} T_{0}} \approx 1.4 \frac{\left(M_{*} / M_{\odot}\right)}{\left(T_{0} / 10^{4} \mathrm{~K}\right)} \mathrm{AU} .
$$

This formula is functionally the same as given in Hollenbach et al. (1994), but differs by a factor of 0.2 (for the case of a monatomic case with $\gamma=5 / 3$ ). An additional non-adiabatic analysis indicates that equation (31) is also true for the isothermal case. Equation (31) may have application to irradiated AGN disks as well as protostellar disks (Woods et al. 1996).

\section{Acknowledgments}

We wish to acknowledge the support from the CSIRO/MIT (grant CZ-25), the Monash University Department of Mathematical Sciences (sections CSPA and $\mathrm{MC}^{2}$ ), the Australian Academy of Sciences travel grant scheme, and the Hawaii Institute of Geophysics and Planetology.

\section{References}

Begelman, M. C., McKee, C. F., \& Shields, G. A. 1983, ApJ 271, 70 Hollenbach, D., Johnstone, D., Lizano, S., \& Shu, F. 1994, ApJ, 428,654

Woods, D. T., Klein, R. I., Castor, J. I., McKee, C. F., \& Bell J. B. 1996, ApJ, 461, 767 